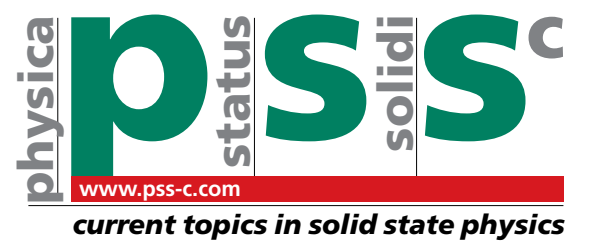

\title{
Hot carrier cooling by acoustic phonons in epitaxial graphene by ultrafast pump-probe spectroscopy
}

\author{
Dong Sun ${ }^{1}$, Charles Divin ${ }^{1}$, Claire Berger ${ }^{2}$, Walt A. de Heer², Phillip N. First ${ }^{2}$, and Theodore B. Norris ${ }^{*} 1$ \\ ${ }^{1}$ Center for Ultrafast Optical Science, University of Michigan, 2200 Bonisteel Blvd., 48109 Ann Arbor, USA \\ ${ }^{2}$ School of Physics, Georgia Institute of Technology, 30332 Atlanta, USA
}

Received 4 October 2010, revised 10 January 2011, accepted 10 January 2011

Published online 21 February 2011

Keywords graphene, acoustic phonons, hot carrier cooling, pump-probe spectroscopy

* Corresponding author: e-mail tnorris@umich.edu, Phone: +001 734764 9269, Fax: +001 7347634876

We report the application nondegenerate ultrafast midinfrared spectroscopy to investigate the acoustic phonon cooling process in epitaxial graphene. We show that the powerdependent experimental results match theoretical predictions of the low temperature acoustic cooling process. The hot phonon effect in acoustic phonon cooling is observed experimentally, and a deformation potential of $30 \mathrm{eV}$ can be determined from fitting the data.
1 Introduction Graphene grown epitaxially on $\mathrm{SiC}$ substrates is being actively researched as a highly promising platform for high speed carbon-based nanoelectronic and optoelectronic devices [1-3]. In high-speed devices, due to the presence of time-dependent high electric fields, carriers gain energy much faster than they are able to lose energy to the lattice, leading to a significantly elevated electron temperature $\mathrm{T}_{\mathrm{e}}[4]$. As the transport properties at high field are dominated by hot carriers, the understanding of cooling processes of hot carriers in multilayer epitaxial graphene (MEG) through the energy exchange between carriers in adjacent layers, the graphene lattice, and the heat dissipation to the SiC substrate, are crucial to high speed electronic device applications.

Ultrafast pump probe spectroscopy has been proven to be a powerful tool to time resolve the cooling of hot carriers in multilayer epitaxial graphene [5]. During the $100 \mathrm{fs}$ pump pulse excitation, the hot electrons thermalize through sub-30 fs electron-electron scattering [6] and reach a quasi-equilibrium state with electron temperature above $1000 \mathrm{~K}$. Since the electron heat capacity depends on the doping intensity and the pump beam is attenuated by each graphene layer, the initial electron temperature in the top layers of the sample is higher. However, hot electrons can efficiently transfer heat and reach equilibrium between the layers through a very fast interlayer thermal coupling during the first few picoseconds [7]. At the same time, the hot electrons within each layer relax to about $250-300 \mathrm{~K}$ via coupling to graphene optical phonon and possibly $\mathrm{SiC}$ substrate optical phonons [7]. Once the carrier temperature has fallen below about $300 \mathrm{~K}$, the cooling is mainly determined by acoustic phonon emission; this process has been recently theoretically investigated in by Bistritzer and MacDonald [8].

In this paper, we use pump-probe spectroscopy to quantify the carrier cooling and fit the results to the Bistritzer and MacDonald theory. We pump at $800 \mathrm{~nm}$ and probe at $1.88 \mu \mathrm{m}$ probe to study the low temperature acoustic cooling process in multilayer epitaxial graphene. By fitting power dependent low temperature cooling behaviour with rate equation predicted by Bistritzer and MacDonald, we can determine the lattice deformation potential.

2 Experiment Epitaxial graphene samples used in this work are grown by thermal desorption of $\mathrm{Si}$ atom from $\mathrm{SiC}$ substrate [9]. For samples grown on C-terminated face of single-crystal $4 \mathrm{H}-\mathrm{SiC}$, the adjacent graphene layer is electronically decoupled or very weakly coupled to other layers due to the rotational stacking order [10]. The sample used in the pump power dependent measurements reported here (Fig. 1) has 21 layers with the most heavily doped layer having a Fermi level $348 \mathrm{meV}$ above the Dirac point determined from previous ultrafast spectroscopy measurements [5]. The doping density and charge distribution 
of C-face multilayer epitaxial graphene (MEG) samples are determined by assuming the same screening length as found in previous ultrafast spectroscopy measurements on similar samples having 63 layers [11]. Generally, the first few layers are heavily doped by charge transferred from the graphene-SiC interface [12]. The doping density of the subsequent graphene layers decreases, asymptotically approaching approximately $10^{10} \mathrm{~cm}^{-2}$ with charge screening length of 1 layer [11].

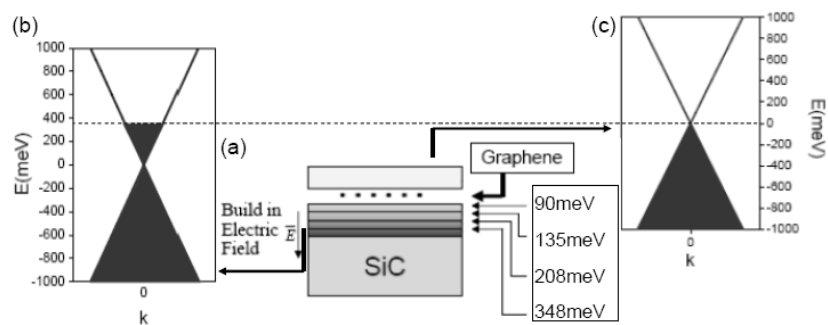

Figure $1^{k}$ Sample structure and energy dispersion curve. (a) Sample structure of multilayer epitaxial graphene. The sample has a buffer layer on the $\mathrm{SiC}$ substrate. The subsequent layers are doped by charge transferred from the graphene-SiC interface. The Fermi levels of the first four layers are labeled. (b), (c) Energy dispersion curves of most heavily doped and undoped graphene layers. The Fermi level is labeled with a dashed line lying $348 \mathrm{meV}$ above the Dirac point of the doped graphene layer and passing through the Dirac point of the undoped graphene layers.

For the infrared probe experiments, a $100-\mathrm{fs} 250-\mathrm{kHz}$ amplified Ti: sapphire laser at $800 \mathrm{~nm}$ pumps an infrared optical parametric amplifier (OPA) with signal wavelength tunable from 1.1 to $1.6 \mu \mathrm{m}$ and idler wavelength tunable from 1.6-2.6 $\mu \mathrm{m}$. The remnant $800 \mathrm{~nm}$ is compressed back below $100 \mathrm{fs}$ and used as the pump. The OPA idler is tuned to $1.88 \mu \mathrm{m}$ and used as probe. The pulse width of the OPA idler at the sample is estimated to be around 150 fs. The pump and probe are co-linearly polarized and focused to about $100-\mu \mathrm{m}$ and $50-\mu \mathrm{m}$ diameter spots respectively on the sample. The probe beam after the sample is detected by an InGaAs photo detector and lock-in amplifier referenced to the $4.2-\mathrm{kHz}$ mechanically chopped pump. The sample temperature is cooled down to $10 \mathrm{~K}$ in a helium flow cryostat. The slight angle between the beams is sufficiently small to prevent temporal walk off across the relatively large probe spot.

3 Experimental result We excite the hot carriers with different initial electron temperatures by varying the 800 $\mathrm{nm}$ pump power, while probing $(1.88 \mu \mathrm{m})$ just below the Fermi level of the first doped layer, but far above the Fermi level of all the other layers. We show below in our transfer matrix simulation that the selection of this specific probe wavelength guarantees that the DT signal at low temperature has the dominant contribution from the most heavily doped layer.

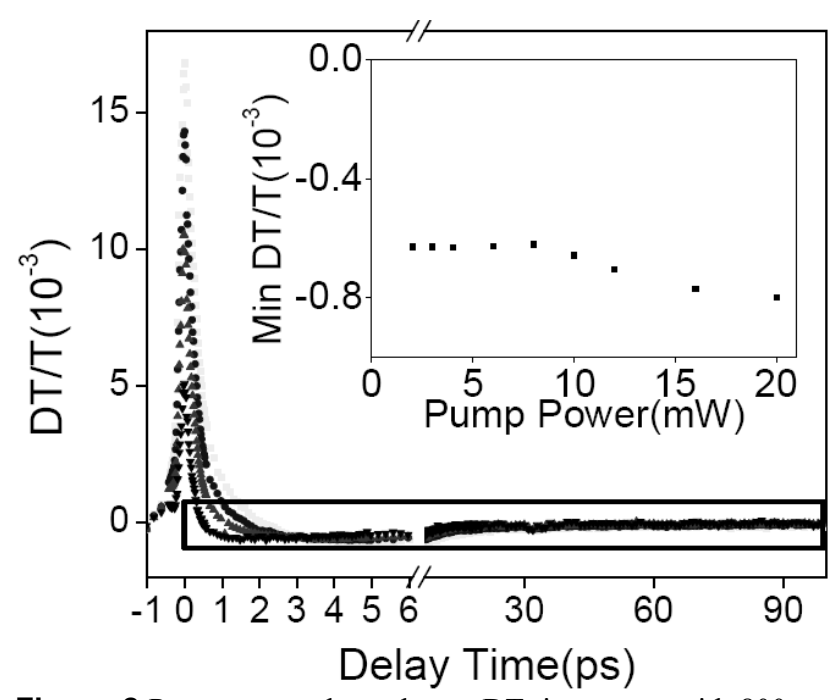

Figure 2 Pump power dependence. DT time scans with 800-nm pump and $1.8-\mu \mathrm{m}$ probe at $10 \mathrm{~K}$ on a 21-layers MEG sample with $3 \mathrm{~mW}$ (down triangle), $6 \mathrm{~mW}$ (up triangle), $10 \mathrm{~mW}$ (dot) and $16 \mathrm{~mW}$ (grey rectangular) pump power respectively. The inset shows the minimum negative DT amplitude at several 800 nm pump powers.

The power-dependent data are shown in Fig. 2. After the initial thermalization and subsequent fast cooling during the first few picoseconds, the DT signal reaches a minimum negative point. Interestingly, as shown in the inset of Fig. 2, the minimum negative DT is almost a constant for relative low pump power excitations and only decreases slightly at high pump power. The DT relaxes to a flat negative value within 10 s of ps delay depending on the pump power; this is a direct result of a higher lattice temperature at higher pump power. The cooling of the graphene lattice occurs finally through heat dissipation to the $\mathrm{SiC}$ substrate. This takes order longer than $100 \mathrm{ps}$ which is beyond the largest experimental measured probe delay [7].

\section{Transfer matrix simulation based on electron} temperature dependent dynamics conductivity The differential transmission signal directly measured by the experiment can be related to the electron temperature of each graphene layer by a transfer matrix calculation. The transfer matrix of a graphene layer relates the incident and reflected probe fields via the dynamical conductivity $\sigma$ of the layer [13]:

$\left[\begin{array}{c}E_{i}^{+} \\ E_{i}^{-}\end{array}\right]=\left[\begin{array}{ll}\frac{1}{2}+\frac{\eta_{i}}{2 \eta_{j}}+\frac{\eta_{i} \sigma}{2} & \frac{1}{2}-\frac{\eta_{i}}{2 \eta_{j}}+\frac{\eta_{i} \sigma}{2} \\ \frac{1}{2}-\frac{\eta_{i}}{2 \eta_{j}}-\frac{\eta_{i} \sigma}{2} & \frac{1}{2}+\frac{\eta_{i}}{2 \eta_{j}}-\frac{\eta_{i} \sigma}{2}\end{array}\right]\left[\begin{array}{l}E_{j}^{+} \\ E_{j}^{-}\end{array}\right]$

where $\eta_{i}=\sqrt{\mu_{i} / \varepsilon_{i}}$ is the dielectric impedance, and $\mathrm{E}_{\mathrm{i}}^{+}$, $\mathrm{E}_{\mathrm{i}}^{-}$are the incident and reflected fields, respectively, and $\mathrm{E}_{\mathrm{j}}^{+}, \mathrm{E}_{\mathrm{j}}^{-}$are the transmitted fields in the forward and backward directions. The conductivity is a function of electron temperature, so the transmission is a function of electron 
temperature. We utilize a model for the conductivity including its dependence on electron temperature $T_{e}$ and layer Fermi level $\mu$ [14]:

$$
\begin{aligned}
& \sigma_{\alpha \beta}^{\text {int } r a}(\omega)=\frac{-i e^{2}}{\hbar^{2}(\omega+i 0) S} \sum_{\vec{k} l} \frac{\partial E_{\vec{k} l}}{\partial k_{\alpha}} \frac{\partial f\left(E_{\vec{k} l}\right)}{\partial E_{\vec{k} l}} \frac{\partial E_{\vec{k} l}}{\partial k_{\beta}} \\
& \sigma_{\alpha \beta}^{\text {inter }}(\omega)=\frac{i e^{2} \hbar}{S} \sum_{\vec{k}, l \neq l^{\prime}} \frac{f\left(E_{\vec{k} l^{\prime}}\right)-f\left(E_{\vec{k} l}\right)}{E_{\vec{k} l^{\prime}}-E_{\vec{k} l}-\hbar(\omega+i 0)} \frac{1}{E_{\vec{k} l^{\prime}}-E_{\vec{k} l}} \\
& \cdot<\vec{k} l\left|\hat{v}_{\alpha}\right| \vec{k} l^{\prime}><\vec{k} l^{\prime}\left|\hat{v}_{\beta}\right| \vec{k} l>
\end{aligned}
$$

Here $E_{\mathbf{k} l}=(-1)^{l} \hbar V k$ is the eigenenergy spectrum for electron $(1=1)$ and hole bands $(1=2)$, and the corresponding wave functions are $|\mathbf{k} l\rangle, \alpha=(x, y), \sigma_{\alpha}$ are Pauli matrixes, $\mathbf{k}=\left(k_{x}, k_{y}\right)$ and $k=\sqrt{k_{x}^{2}+k_{y}^{2}}$ is the wave vector, $f\left(E_{\mathbf{k} l}\right)$ is the Fermi distribution function which is function of $\mathrm{T}_{\mathrm{e}}$ and $\mu, \hat{v}_{\alpha}=V \sigma_{\alpha}$ is the velocity operator, and $\mathrm{S}$ is the sample area.

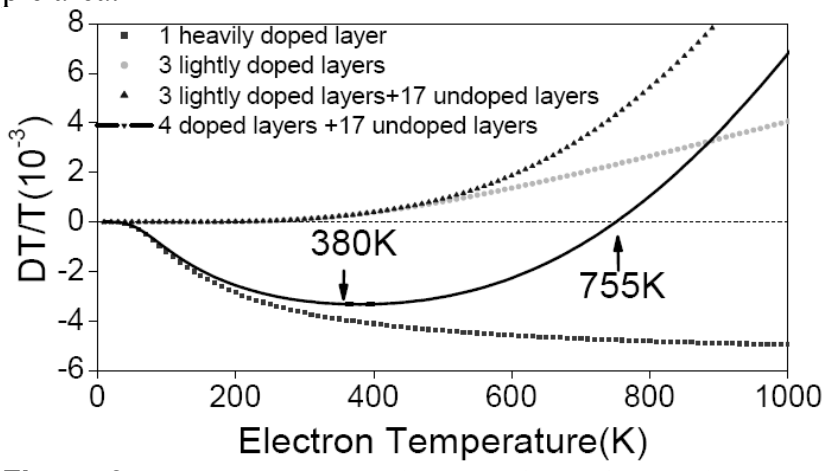

Figure 3 Transfer matrix simulation of the DT/T signal of MEG with different graphene layers combination at different electron temperatures.

The product of all transfer matrices, including that of the substrate, gives the optical response of the MEG sample. In the simulation we include four highly doped layers with charge densities corresponding to those measured in the experiment [11] and we further assume all graphene layers have the same electron temperature following the first few picoseconds initial cooling; this is a reasonable assumption considering the strong interlayer thermal coupling observed in a separate degenerate pump probe experiment [7] A simulation of the DT signal at $1.88-\mu \mathrm{m}$ probe wavelength as a function of electron temperature is shown in Fig. 3. We see that the DT response is dominated by the contribution of the first doped layer at low electron temperatures (below $300 \mathrm{~K}$ ), so the DT tails unambiguously measure the relaxation of the hot carriers in the first doped layer when the electron temperature is below $300 \mathrm{~K}$. This is not surprising since the probe photon energy is very close to the Fermi level of the first doped layer and far above the Fermi level of all other layers.

The simulation results including all layers in the sample show DT zero crossings around $750 \mathrm{~K}$ and a minimum negative DT round $380 \mathrm{~K}$. Therefore when the electron temperature relaxes to $380 \mathrm{~K}$, the DT reaches the same negative DT amplitude regardless of the initial electron temperature at different pump excitations; this matches the experimental observation shown in the inset of Fig. 2. Thus the minimum negative DT is a clear experimental indicator of $380 \mathrm{~K}$ electron temperature.

\section{Electron and lattice heat capacity in graphene}

The electron heat capacity of the doped layer and undoped layer at relatively low electron temperature can be obtained from a simple statistical mechanical calculation. For undoped layers, the electron heat capacity has $\mathrm{T}_{\mathrm{e}}^{2}$ dependence:

$C_{e}\left(T_{e}\right)=\frac{\partial E}{\partial T_{e}}=\frac{18 \zeta_{3}}{\pi} k_{B}\left(\frac{k_{B} T_{e}}{\hbar v_{F}}\right)^{2}$

where $\mathrm{T}_{\mathrm{e}}$ is the electron temperature, $\mathrm{v}_{\mathrm{F}}$ is the Fermi velocity, $\mathrm{k}_{\mathrm{B}}$ is the Boltzmann constant and $\zeta_{3}=\int_{0}^{\infty} d x \frac{x^{2}}{e^{x}+1}$ is the Riemann zeta function, so for the undoped layers: $C_{e}^{\text {undoped }}\left(T_{e}\right)=8.136588 * 10^{-13} T_{e}^{2}\left(\mathrm{~J} / \mathrm{m}^{2} \mathrm{~K}\right)$

For doped layers, the electron heat capacity has $\mathrm{T}_{\mathrm{e}}$ dependence:

$C_{e}\left(T_{e}\right)=\frac{1}{A} \frac{\partial U}{\partial T}=\frac{2 \pi}{3} \frac{E_{F}^{0}}{\hbar^{2} v_{F}^{2}} k_{B}^{2} T_{e}$,

where $E_{F}$ is the Fermi level of the doped layer, for the heavily doped layer with Fermi level of $350 \mathrm{meV}$, $C_{e}^{\text {doped }}\left(T_{e}\right)=2 * 10^{-9} T_{e}\left(\mathrm{~J} / \mathrm{m}^{2} \mathrm{~K}\right)$. At low temperature the lattice heat capacity is dominated by the acoustic phonons, in our simulation, we use a simple linear temperature dependent lattice heat capacity: fit from Mounet and Marzari's first principle calculation [15]:

$$
C_{l}\left(T_{l}\right)=1.3 * 10^{-6} T_{l}\left(J / m^{2} K\right)
$$

\section{Fitting of low temperature acoustic phonon} cooling Carrier cooling through acoustic phonon emission has been treated theoretically by R. Bistritzer and D. MacDonald [8] for both lightly- and heavily-doped graphene layers. In the neutral regime, the electron temperature is described by $\partial_{t} T_{e}=-\gamma T_{e}^{2}\left(T_{e}-T_{l}\right)$, where $\mathrm{T}_{\mathrm{e}}$ and $\mathrm{T}_{1}$ are electron and lattice temperature respectively, $\gamma=1.18 * 10^{3} \mathrm{D}^{2}\left(\mathrm{meV}^{2} \mathrm{~s}\right)^{-1}$ and $\mathrm{D}$ is the deformation potential measured in $\mathrm{eV}$. This equation applies to the large number of undoped layers in our multilayer epitaxial graphene sample at low carrier temperature. In contrast, the electron temperature in the heavily doped bottom layers follows $\partial_{t} T_{e}=-\gamma_{d}\left(T_{e}-T_{l}\right) / T_{e}$, where $\gamma_{d}=0.133 D^{2} n^{3 / 2} \mathrm{meV} / \mathrm{ns}$ with $\mathrm{n}$ being measured in units of $10^{12} \mathrm{~cm}^{-2}$. This predicts an acoustic phonon cooling time on the order of $10 \mathrm{ps}$ in the heavily doped layer which matches the measurements shown in Fig. 2. 
Since the heat capacity of the undoped layer is at least an order of magnitude smaller than that of the heavily doped layer at electron temperatures below $380 \mathrm{~K}$, the effect of the interlayer thermal coupling on the cooling of the highly doped layer can be safely ignored in the simulation. The slow ns thermal coupling to the $\mathrm{SiC}$ substrate during the $10-40$ ps acoustic cooling process was ignored in the simulation, and a constant lattice temperature was used in this low electron temperature cooling simulation due to the 3 orders larger lattice heat capacity compared to the electron heat capacity (the lattice temperature was estimated by assuming $2.3 \%$ of the pump energy is deposited to the electron gas in each graphene layer and then is transferred to the lattice). The ratio of energy loss via optical phonons to the energy loss vai the acoustic phonons for undoped and heavily doped layers at different carrier temperatures has been calculated by Bistritzer and MacDonald [8]. For the heavily doped layer, the optical phonon cooling shuts down completely at $250 \mathrm{~K}$ from their simulation, and at an electron temperature of $380 \mathrm{~K}$, this ratio is below $20 \%$. In our simulations, the energy relaxation through optical phonon between $250 \mathrm{~K}$ and $380 \mathrm{~K}$ is ignored; this will make the simulation results slower than the experiment in this temperature range. Another important process that is not included in our simulation is the decay of the hot optical phonon to the acoustic phonon mode in the first few ps, the life time of optical phonon in graphene has been measured to be 2.5 ps [16]. This contribution accounts for the relatively slower simulated cooling at high electron temperature before the decay of optical phonons and relative faster simulated cooling when all optical phonons decays to acoustic phonons.

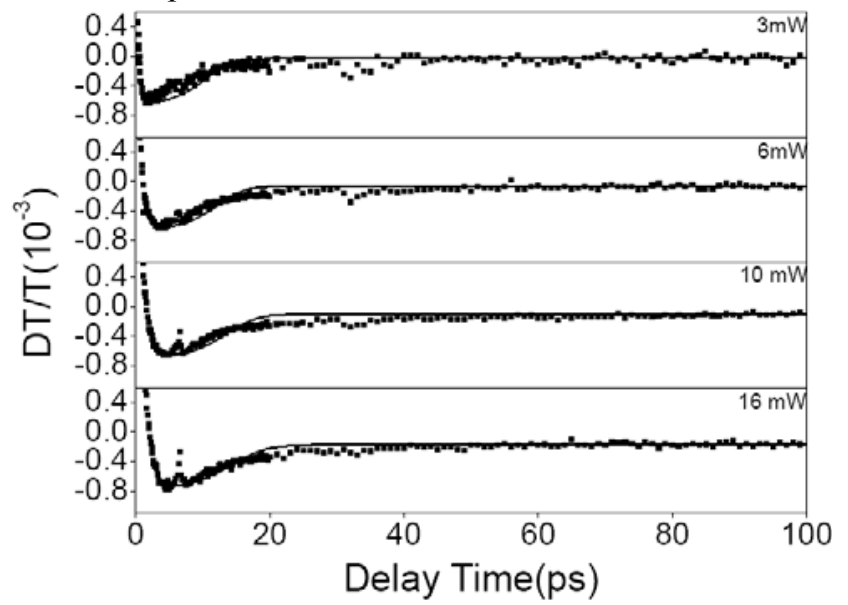

Figure 4 Acoustic phonon cooling fitting. The measured DT signal (dots) at low temperature is plotted along with the theoretical acoustic phonon cooling model (solid).

Figure 4 shows a fit of the low energy acoustic phonon cooling process in the heavily doped layers starting from $380 \mathrm{~K}$ which can be identified from the minimum negative DT in the experimental differential transmission curve. Besides the secondary peaks at $\sim 6$ ps and $\sim 30$ ps due to back reflection of pump pulse in $\mathrm{SiC}$ substrate and $\mathrm{CaF}_{2}$ crystat windows, the experimental result is well fit with a deformation potential of $\sim 30 \mathrm{eV}$ which is within the 20 and $50 \mathrm{eV}$ range bounded by transport measurements [17].

7 Conclusion We have applied pump power dependent nondegenerate ultrafast infrared spectroscopy to investigate the acoustic phonon cooling process in the most heavily doped layer in multilayer epitaxial graphene. We show that the experimental differential transmission recovery can be well fit by the theoretically predicted low temperature acoustic cooling process. We also determine the deformation potential to be $30 \mathrm{eV}$ from our experiment.

Acknowledgements This project has been supported by NSF MRSEC 0820382.

\section{References}

[1] Y. Zhang et al., Nature 459, 820 (2009).

[2] W. A. de Heer et al., Solid State Commun. 143, 92 (2007).

[3] C. Berger et al., J. Phys. Chem. B 108, 19912 (2004).

[4] O. G. Balev, F. T. Vasko, and V. Ryzhii, Phys. Rev. B 79, 165432 (2009).

[5] D. Sun et al., Phys. Rev. Lett. 101, 157402 (2008).

[6] M. Breusing, C. Ropers, and T. Elsaesser, Phys. Rev. Lett. 102, 086809 (2009).

[7] D. Sun et al., to be submitted 2010.

[8] R. Bistritzer and A. H. MacDonald, Phys. Rev. Lett. 102, 206410 (2009).

[9] C. Berger et al., Science 312, 1191 (2006).

[10] J. Hass et al., Phys. Rev. Lett. 100, 125504 (2008).

[11] D. Sun et al., Phys. Rev. Lett. 104, 136802 (2010).

[12] F. Varchon et al., Phys. Rev. Lett. 99, 126805 (2007).

[13] M. Born, and E. Wolf, Principles of Optics (Cambridge University Press, 1999).

[14] S. A. Mikhailov and K. Ziegler, Phys. Rev. Lett. 99, 016803 (2007).

[15] N. Mounet and N. Marzari, Phys. Rev. B 71, 205214 (2005).

[16] H. Wang et al., Appl. Phys. Lett. 96, 081917.

[17] K. I. Bolotin et al., Phys. Rev. Lett. 101, 096802 (2008). 